\title{
Tecnologias emissoras de radiação ionizante e a necessidade de educação permanente para uma práxis segura da enfermagem radiológica
}

\author{
Issuing technologies of ionizing radiation and the need of permanent \\ education for a safe praxis in radiological nursing \\ Tecnologías emisoras de radiación ionizante y la necesidad de la educación \\ permanente para una praxis segura de la enfermería radiológica
}

\section{Rita de Cássia Flôr', Francine Lima Gelbcke}

'Universidade Federal de Santa Catarina. Programa de Pós-Graduação em Enfermagem. Florianópolis, SC

Submissão: 12/12/2008

Aprovação: 3 l/08/2009

\section{RESUMO}

Refletir acerca das tecnologias radiológicas e da necessidade da educação permanente Que aborde temas relacionados com a aplicação das radiações ionizantes na práxis da enfermagem radiológica é mister, haja vista o avanço tecnológico Que revolucionou as práticas em saúde e conseQüentemente a assistência de enfermagem, pois essas tecnologias incidem fortemente sobre o trabalho e a Qualificação do trabalhador. Esta reflexão discorre sobre as áreas de atuação da enfermagem radiológica nos Serviços de Radiologia e Diagnóstico por Imagem (SRDI), assim como a necessidade da educação permanente para a práxis segura nessa especialidade. Apresenta estudos de alguns autores acerca dessas necessidades, principalmente no Que tange à proteção radiológica e à necessidade de Qualificação desses trabalhadores.

Descritores: Trabalho; Tecnologia radiológica; Enfermagem; Educação continuada.

\begin{abstract}
Reflecting about radiological technologies and the need for permanent education to address issues related to the application of ionizing radiation on radiological nursing practice is of a great importance, due to the technological progress that revolutionized practices in health and, consequently, the nursing care. So, these technologies strongly focus on labor and worker's Qualification. This reflection happens in the acting areas of radiological nursing in the Radiology Services and Image Diagnosis (SRDI), as well as the need for permanent education to the safe praxis on this specialty which is presented through some authors' studies. Primarily, in relation to the radiological protection and need to Qualify these workers.
\end{abstract}

Descriptores: Work; Radiologic technology; Nursing; Continuous education.

\section{RESUMEN}

Reflexionar acerca de las tecnologías radiológicas y la necesidad de una educación permanente Que aborde temas relacionados con la aplicación de radiaciones ionizantes en la praxis de la enfermería radiológica es fundamental, en virtud del avance tecnológico Que revolucionó las prácticas en la salud y, consecuentemente, la asistencia de la enfermería, pues esas tecnologías inciden fuertemente sobre el trabajo y la calificación del trabajador. Esta reflexión discurre sobre las áreas de actuación de la enfermería radiológica en los Servicios de Radiología y Diagnóstico por Imagen (SRDI), así como la necesidad de una educación permanente para la praxis segura en esta especialidad, de la misma forma que los estudios de algunos autores son presentados en relación a estas necesidades; principalmente en lo Que se refiere a la protección radiológica y a la necesidad de calificación de esos trabajadores.

Descriptores: Trabajo; Tecnología radiológica; Enfermería; Educación continua. 


\section{INTRODUÇÃO}

Este artigo parte do pressuposto de Que é fundamental refletir acerca das tecnologias radiológicas e da necessidade da educação permanente com foco na aplicação das radiações ionizantes na práxis da enfermagem radiológica, haja vista o avanço tecnológico Que revolucionou as práticas em saúde e, conseQüentemente, a assistência de enfermagem e o modo como essas tecnologias incidem fortemente sobre o trabalho e a Qualificação do trabalhador.

A enfermagem radiológica é a especialidade da enfermagem relacionada ao cuidado do usuário submetido a procedimentos diagnósticos e terapêuticos nos Serviços de Radiologia e Diagnóstico por Imagem (SRDI) ${ }^{(1)}$.

Os SRDI, em especial na práxis da enfermagem radiológica, vem sofrendo grandes transformações. Isso porque, desde sua descoberta (por Roentgen, em 1895), a radiação X tem sido empregada em grande escala na área da saúde para fins terapêuticos e diagnósticos, em contribuição para o desenvolvimento tecnológico da área da saúde ${ }^{(2-4)}$.

Após a descoberta dos raios X, outros métodos de obtenção de imagens foram surgindo, entre eles as tecnologias chamadas de alta complexidade, Que demandam equipamentos sofisticados e trabalhadores Qualificados. O equipamento de raios X já foi considerado tecnologia de alta complexidade. Hoje o Que tem esse status são os equipamentos de medicina nuclear, mamografia, tomografia computadorizada, hemodinâmica, ressonância magnética e, mais recentemente, tomografia com emissão de pósitrons ${ }^{(4)}$. Além dessas especialidades, também fazem parte da práxis da enfermagem radiológica os serviços de radiologia convencional, radioterapia (braquiterapia e teleterapia), entre outras áreas de aplicações da radiação ionizante na saúde.

É importante ressaltar que os novos equipamentos e procedimentos não substituem os antigos métodos, porQue não se trata de uma mudança da forma de prestar o serviço, mas da criação de um novo tipo de serviço. Isso ocorre particularmente "no campo do diagnóstico das doenças, em Que a cada dia surgem inovações tecnológicas”(5) e conseqüentemente a necessidade de Qualificar o trabalhador para essa nova demanda.

Pelo fato de a enfermagem radiológica atuar cada vez mais com tecnologias emissoras de radiação ionizante em seu processo de trabalho, é imperiosa sua Qualificação, haja vista Que esse campo de atuação profissional tende cada vez mais a aumentar e a enfermagem precisa preparar-se para atuar com segurança nessas especialidades.

Nesse sentido, esta reflexão tem o propósito de chamar a atenção dos trabalhadores de enfermagem para a práxis da enfermagem radiológica com as tecnologias emissoras de radiação ionizante nos SRDI e para a importância da educação permanente Que aborde temas atinentes a esse processo de trabalho.

\section{TECNOLOGIAS EMISSORAS DE RADIAÇÃO IONIZANTE E O CAMPO DE ATUAÇÃO DA ENFERMAGEM RADIOLÓGICA}

Das tecnologias emissoras de radiação ionizante serão aQui detalhadas as áreas de maior demanda de procedimentos envolvendo diretamente os trabalhadores de enfermagem. Antes, porém, é oportuno esclarecer o conceito de radiação ionizante. Considera-se radiação ionizante QualQuer partícula ou radiação eletromagnética Que, ao interagir com a matéria, "arranca" elétrons dos átomos ou de moléculas, transformando-os em íons, direta ou indiretamente. Assim, as partículas alfa e beta e a radiação gama, emitidas por fontes radioativas, bem como os aparelhos de raios $\mathrm{X}$, são consideradas radiações ionizantes ${ }^{(6)}$.

Entre as suas especialidades, a radiologia e o diagnóstico por Imagem exigem grande variedade de procedimentos relacionados ao uso de radiação ionizante e à atuação dos trabalhadores de enfermagem. A principal delas é a radiologia convencional, Que utiliza os exames de radiografia, sendo as mais conhecidas as radiografias de tórax e de membros superiores e inferiores. A ação da enfermagem nessa especialidade ocorre principalmente no preparo dos usuários em exames contrastados (ou seja, na administração das soluções por via parenteral, oral e retal), na orientação antes e após os exames, no preparo do ambiente e dos materiais a serem utilizados ${ }^{(7.8)}$.

A tomografia computadorizada (TC) é outra especialidade com alta demanda de solicitação de exames, em Que os trabalhadores de enfermagem prestam assistência antes, durante e após os exames. Também envolve preparo e administração de contrastes, entretanto, nesse caso, existe a possibilidade de o contraste ser introduzido por bombas injetoras. Além disso, faz parte da atuação da enfermagem o acompanhamento de alguns exames, especialmente aQueles em Que os usuários não podem ficar sozinhos na sala de exame devido às suas condições clínicas - é o caso dos politraumatizados, das crianças, entre outros. O acompanhamento desses exames requer que os trabalhadores de enfermagem sejam orientados, principalmente acerca de sua proteção radiológica, de modo a evitar as exposições desnecessárias às radiações ${ }^{(7,8)}$.

A medicina nuclear $(\mathrm{MN})$ constitui outra especialidade dessa área do conhecimento. Diferentemente dos exames anteriores, caracteriza-se pela exposição à radiação, pois nela existe o perigo constante de contaminação radioativa e não de irradiação. É importante esclarecer a diferença entre contaminação e irradiação(?) .

A contaminação radioativa se caracteriza pela presença indesejável de um material em determinado local. A irradiação é a exposição de objeto ou corpo à radiação; irradiar, portanto, não significa contaminar. lá contaminar com material radioativo implica irradiar o local onde se encontra esse material. Na medicina nuclear os trabalhadores encontram-se mais vulneráveis às radiações ionizantes, porQue nesses ambientes os usuários Que necessitam desse tipo de tratamento se tornam fontes radioativas ao receberem os radiofármacos para a realização dos exames ${ }^{(9)}$.

Na maioria das vezes, esses radiofármacos são aplicados pelos trabalhadores de enfermagem, pois sua prática envolve conhecimento de fundamentos de enfermagem, como preparo e administração de medicações parenterais e orais ${ }^{(8)}$. O trabalho em um serviço de medicina nuclear necessita de uma equipe multiprofissional, pois envolve múltiplos saberes. Tal equipe geralmente é composta de médico especialista em medicina nuclear, radiofarmacêutico, físico, enfermeiro, técnico em medicina nuclear e técnicos em radiologia e em enfermagem. Entre as principais atribuições dessa equipe multiprofissional, são específicas da equipe de enfermagem: administração da dose recomendada de radiofármaco; orientação Quanto aos procedimentos a serem realizados, incluindo os controles e liberação dos usuários internados; agendamento dos exames preliminares; coleta de sangue para dosagem hormonal; controle e 
administração da medicação prescrita; orientação Quanto à internação e alta; e atendimento de imediato às eventuais intercorrências clínicas ${ }^{(10-12)}$

A radioterapia é outra área de atuação da enfermagem. Ao contrário do Que ocorre na medicina nuclear, os usuários irradiados para o tratamento com radioterapia não ficam radioativos, e assim os trabalhadores de enfermagem podem prestar assistência, desde Que façam uso de medidas de proteção radiológica apropriadas. O tratamento radioterápico possui, basicamente, dois grandes grupos: teleterapia e braquiterapia ${ }^{(10,11)}$.

Na teleterapia, tele significa "distância". Nessa categoria encuadram-se os feixes de raios $\mathrm{X}$, os feixes de raios gama e os elétrons de alta energia. Esses feixes "são utilizados através de dispositivo, contendo em seu interior uma fonte de radiação Que, colocados a aproximadamente um metro do usuário, promovem a irradiação"(13).

Na braquiterapia, "braqui significa curto, perto, é um método de terapia no Qual uma ou várias fontes encapsuladas são utilizadas para liberar radiação alfa ou beta, a uma distância de poucos centímetros, através de aplicações intersticiais, intracavitárias ou superficiais"(13). Durante a aplicação, essa fonte emite radiação de dentro para fora. Os trabalhadores Que prestam assistência não devem permanecer muito tempo nas proximidades devido à possibilidade de exposição. Removida a fonte, nada fica radioativo ${ }^{(13,14)}$.

Estudos relatam experiências com braquiterapia intracavitária na neoplasia uterina, nas Quais foram apresentados protocolos clínicos e físicos dos procedimentos executados, destacando-se a importância do treinamento específico Quanto às medidas de proteção e a obediência aos rigorosos princípios da proteção radiológica na assistência de enfermagem. Referem também Que a enfermagem participa em todos os procedimentos realizados com usuários submetidos a esse tratamento, incluindo a retirada e colocação da fonte radioativa. Reforçam ainda a necessidade de toda equipe de saúde possuir noções básicas de proteção radiológica e usar dosímetros individuais de radiação ${ }^{(14)}$.

Em um serviço de braquiterapia, a equipe de trabalho é multidisciplinar, e $80 \%$ dela é composta de trabalhadores de enfermagem Que participam ativamente da assistência prestada aos usuários braquiterápicos. Esses autores enfatizam, porém, Que, apesar de esses trabalhadores representarem significativamente o maior contingente na área, eles não possuem conhecimentos de proteção radiológica e de como se operacionalizam os equipamentos de segurança existentes. Chegam a sugerir programa de treinamento de proteção radiológica para os trabalhadores de enfermagem ${ }^{(14)}$.

Estudos vêm mostrando a importância dos trabalhadores de enfermagem no preparo do usuário para o tratamento de radioterapia. Reforçam Que o enfermeiro especialista em radioterapia deve buscar conhecimentos teórico-práticos sobre o tratamento em teleterapia, braQuiterapia e radioproteção, por meio de cursos de atualização, reuniões científicas, participação em eventos, entre outros ${ }^{(12-14)}$

É evidenciada também a necessidade de se manter uma educação permanente para esclarecimento aos profissionais Que se expõem à radiação ionizante, não só por meio de fornecimento de equipamentos, mas também mediante controle e validação dos procedimentos de proteção, tanto para a equipe de saúde como para os pacientes ${ }^{(13)}$.

O enfermeiro no serviço de radioterapia é visto como um profissional diferenciado e Que necessita de conhecimentos especializados e habilidades específicas para desenvolver suas atividades. No entanto, são poucos os serviços Que valorizam o enfermeiro especializado nessa área do conhecimento e dispõem dele ${ }^{(14)}$.

Outra área de aplicação da radiação envolvendo a atuação da enfermagem é a irradiação de sangue com raios gama. Esse método é usado nos hemocentros, no sangue a ser ministrado em usuários com deficiência imunológica, e diminui a Quantidade de linfócitos T (células de defesa) no sangue doado, o Que reduz em muito o risco de rejeição do órgão ou dos tecidos transplantados por parte do usuário ${ }^{(15)}$.

E, por fim, temos a radiologia intervencionista, Que compreende a hemodinâmica, a neurorradiologia e a radiologia vascular periférica. A radiologia intervencionista é uma especialidade da RDI Que comporta tecnologias modernas utilizadas no diagnóstico e tratamento das patologias, por meio de imagens concebidas por técnicas fluoroscópicas ${ }^{(16)}$.

Em todas essas atividades, o trabalhador deve utilizar pelo menos avental de chumbo e protetor de tireóide para sua proteção. Esses aventais de chumbo $(\mathrm{Pb})$ são confeccionados com equivalência de $0,25 \mathrm{~mm} \mathrm{~Pb}$ a $0,50 \mathrm{~mm} \mathrm{~Pb}$, em razão da necessidade da proteção radiológica. Assim como os aventais, os demais equipamentos individuais plumbíferos, como luvas, óculos e saiotes, também apresentam as mesmas equivalências. Além desses equipamentos, recomenda-se também o uso de dosímetro.

O dosímetro, ou monitor individual, é utilizado pelo trabalhador para medir a dose efetiva de radiação durante o período em Que o trabalhador permanecer em áreas controladas ou durante sua jornada de trabalho. Deve ser utilizado na região mais exposta do tronco. A Portaria 453/1998 instrui Que, durante a utilização de avental plumbífero, o dosímetro deve ser colocado sobre o avental. Recomenda também Que a dose efetiva média anual não deve exceder a $20 \mathrm{mSv}$ (milisievert) em Qualeuer período de cinco anos consecutivos, nem exceder a $50 \mathrm{mSv}$ em nenhum ano. Esclarece ademais Que a dose equivalente anual não deve exceder a $500 \mathrm{mSv}$ para extremidades e a $150 \mathrm{mSv}$ para o cristalino ${ }^{(17)}$.

\section{A EDUCAÇÃO PERMANENTE COMO ESTRATÉGIA PARA A ATUAÇÃO DA PRÁXIS DA ENFERMAGEM RADIOLÓGICA}

$\mathrm{Na}$ área da saúde, especificamente na enfermagem, a busca por um processo educativo contínuo tem sido constante, no sentido de garantir o cuidado nas mais diversas especialidades de enfermagem - neste caso, na enfermagem radiológica. Percebe-se Que essa também é uma preocupação do Ministério da Saúde, no momento em Que instituiu a Política Nacional de Educação Permanente em Saúde (Portaria no 1 98/04GM/MS) como estratégia do Sistema Único de Saúde para a formação e o desenvolvimento dos trabalhadores da saúde e com a Portaria 1996 GM/MS de 20/08/07, Que revogou a Portaria $198 \mathrm{GM} / \mathrm{MS}$ de 13/02/04 ${ }^{(18.19)}$.

Compreende-se Educação Permanente (EP) como uma possibilidade pedagógica no setor da saúde para efetuar relações orgânicas entre ensino e serviço, entre docência e atenção à saúde, além das relações entre formação e gestão setorial, desenvolvimento institucional e controle social em saúde. Dessa forma, a Educação Permanente é aprendizagem no trabalho, onde o aprender e o ensinar se incorporam ao cotidiano das organizações e ao trabalho. Tem como pressuposto pedagógico Que as práticas sejam definidas por múltiplos 
fatores e Que a aprendizagem dos adultos deva ser uma aprendizagem significativa, isto é, Que faça sentido para os sujeitos envolvidos, objetivando a transformação das práticas. Deve acontecer no cotidiano das pessoas e das organizações, a partir de problemas enfrentados na prática $^{(19)}$.

Entretanto, apesar de a EP estar bem fundamentada teoricamente, ainda não se conseguiu trazer para a prática dos serviços seus pressupostos pedagógicos e metodológicos. Esse fato é observado nos Serviços de Radiologia e Diagnóstico por Imagem, pois estes possuem saberes e práticas específicas no seu processo de trabalho e, mesmo sendo setores com características especiais, pouca ênfase é dada a esse conhecimento específico ${ }^{(20)}$.

Há necessidade de se manter uma educação permanente para esclarecimento dos trabalhadores Que se expõem à radiação ionizante, não só por meio de fornecimento de equipamentos, mas também mediante controle e validação dos procedimentos de proteção, tanto para a equipe de saúde como para os usuários.

Aliados à política de EP temos os pactos de gestão do Sistema Único de Saúde, Que consideram a política de recursos humanos para o SUS como um eixo estruturante para a redução dos conflitos, a valorização dos trabalhadores da saúde e a humanização das relações de trabalho. Além disso, o eixo de gestão do trabalho prevê a capacitação e Qualificação dos gestores e técnicos na perspectiva do fortalecimento do trabalho em saúde. Entretanto, mesmo tendo políticas indutoras para a formação e capacitação dos trabalhadores da saúde, o que vemos em nosso cotidiano é o "esquecimento" desse serviço no Que tange à formação e capacitação pelos órgãos formadores e, até mesmo, pelos serviços de saúde em Que estão inseridos ${ }^{(21)}$.

As pesquisas evidenciam a necessidade de um número maior de trabalhadores da área da saúde Que dominem conhecimentos e estejam aptos a manipular e aplicar materiais radioativos nos SRDI. Os aspectos do desenvolvimento seguro de atividades de enfermagem em rotinas de internação em Quartos terapêuticos estão entre os fatores observados nas pesquisas. Além disso, os alertas sobre a importância do treinamento das equipes envolvidas nesse processo de trabalho, promovendo melhor inter-relacionamento dos usuários e dos acompanhantes durante o tratamento naQuele ambiente, levam à conclusão de Que a maioria desses trabalhadores de enfermagem conhece muito pouco sobre o uso dessa tecnologia(22).

Assim, seria prudente que os trabalhadores tivessem no mínimo uma formação básica e experiência necessária para desempenhar seus trabalhos com segurança, sobretudo em relação à exposição aos materiais radioativos, tais como as fontes não seladas ${ }^{(21)}$.

Em 2002, o tema proteção radiológica teve sua primeira conferência internacional dedicada exclusivamente à proteção radiológica ocupacional, cujo mote central foi "protegendo o trabalhador das exposições à radiação ionizante" e Que ocorreu em agosto de 2002, na sede da Organização Internacional do Trabalho (OIT), na cidade de Genebra - Suíça ${ }^{(2)}$.

O resultado dessa conferência mostrou a necessidade de otimização das práticas radiológicas, sendo aspecto fundamental a programação do controle radiológico dos trabalhadores, Que deve ser consolidado por meio de redes de informações, tal como atualmente ocorre com o Programa Internacional de Informações sobre Doses Ocupacionais - ISOE, para as centrais nucleares. Recomendou-se concentrar os esforços nos casos de doses individuais acima de I
$\mathrm{mSv}$ (milisievert) mês ${ }^{(2)}$

Outro tema debatido foi a necessidade de se manter a educação permanente para assegurar boas práticas de segurança radiológica. Além disso, foi recomendado Que as instituições Que empregam radiação ionizante facilitem o acesso dos trabalhadores a cursos de treinamento nessa área do conhecimento, assim como o preparo de materiais educativos elaborados por profissionais Qualificados, sendo mencionada a figura do docente, por ser um profissional melhor preparado para ministrar esse tipo de treinamento.

E, por fim, recomendou-se a implementação de um plano de ação internacional por organismos internacionais, em particular o Organismo Internacional de Energia Atômica (OIEA) e a Organização Internacional do Trabalho (OIT), para a concretização das recomendações de outras medidas substanciais Que permitam reforçar globalmente a proteção radiológica ocupacional ${ }^{(2)}$.

Invisível e atuando de forma lenta, a radiação ionizante causa danos à saúde Quando não são respeitadas rigidamente as precauções para se evitarem as exposições desnecessárias. Nesse sentido, a proteção radiológica constitui importante ferramenta na promoção da saúde dos trabalhadores Que exercem suas atividades com radiação ionizante e, nesse caso, a educação permanente pode contribuir para a melhoria desse processo de trabalho, pois conhecendo o mecanismo de produção, assim como da interação da radiação, os trabalhadores de saúde podem fazer uso das radiações ionizantes em seu processo de trabalho de forma mais otimizada.

Por fim, destaca-se a Resolução do Conselho Federal de Enfermagem - COFEn/211, Que dispõe sobre a atuação dos profissionais de enfermagem Que trabalham com radiação ionizante. Essa resolução apresenta em seu escopo a necessidade de regulamentar as normas de radioproteção e assegurar condições adequadas de trabalho para profissionais de enfermagem que trabalham em radioterapia, medicina nuclear e em outros serviços de radiodiagnóstico. Ainda menciona as competências já estabelecidas na lei do exercício profissional e estabelece as competências específicas do enfermeiro e dos profissionais de nível médio, referentes à atuação com os usuários submetidos à radiação ionizante $\mathrm{e}^{(23)}$.

Todos esses estudos reafirmam nossa preocupação em Que os trabalhadores de enfermagem Que exercem a práxis radiológica o façam de forma consciente, e a educação permanente é o caminho para Que os trabalhadores possam prevenir-se dos danos causados pelos equipamentos emissores de radiação ionizante, haja vista o desenvolvimento de novos equipamentos de tecnologia de ponta como instrumentos de trabalho.

\section{CONSIDERAÇÕES FINAIS}

Refletir acerca da práxis da enfermagem radiológica e suas implicações relativas ao avanço tecnológico dos equipamentos emissores de radiação ionizante permitiu vislumbrar a possibilidade de a enfermagem radiológica se consagrar como uma especialização, a exemplo de outras especialidades da enfermagem. Contudo, existe ainda um longo caminho a ser percorrido, pois a enfermagem, apesar de ter seu campo de trabalho ampliado nessa área, ainda não se deu conta da especificidade do cuidado prestado aos usuários submetidos a diagnóstico ou tratamento utilizando radiação ionizante. Esses aspectos são diferenciados do cuidado prestado pela enfermagem de 
uma forma geral, portanto há necessidade de o trabalhador desenvolver competências específicas, como saber orientar usuários, familiares e demais trabalhadores acerca de como se proteger das radiações ionizantes.

Sabemos Que as rigorosas medidas de proteção radiológica influenciam o cotidiano da assistência de enfermagem, tornando-o desafiador. Nesse sentido, gerenciar a assistência de enfermagem requer oferecer informações relativas a esses cuidados, para Que o usuário se sinta acolhido e o trabalhador protegido. Assim, faz-se necessário Que esses trabalhadores se apropriem desses conhecimentos, tanto para proteger-se como para prestar o cuidado de forma otimizada, e não temorizada, pois Quem trabalha com radiação deve seguir normas rígidas de proteção radiológica, ou seja, a radiação deve ser respeitada, e não temida.

Observamos também Que a capacitação das práticas nos serviços de RDI se dá no âmbito do trabalho mais Que na escola. Assim, a adoção dos princípios da Educação Permanente surge como uma possibilidade para a capacitação desses trabalhadores, já Que, por meio da educação e da reflexão do cotidiano, estabelece-se a possibilidade de um novo agir, criando-se no trabalho um espaço de ação e reação, de escolhas, de um caminho mais seguro para o fazer da enfermagem radiológica.

\section{REFERÊNCIAS}

1. Goodhart I, Page J. Orthopaedic nursing. Radiol Nurs 2007; 26(I): 36-9.

2. Massera G. Algunas conclusiones de la Conferencia Internacional sobre Protección Radiológica Ocupacional. Seguridad radiologica. Rev Sociedad Argent Radioprotección 2003; 28-30.

3. Matushita JPK. História da radiologia. Bol Col Bras Radiol 2002; 168:16-7.

4. Rezende IMO. Uso da tecnologia no diagnóstico médico e suas conseQüências. Ética Rev 2006; (4): 1 8-2 I.

5. Nogueira RP. A força de trabalho em saúde. In: Médice AC, organizador. Textos de apoio: Planejamento I. Recursos humanos em saúde. Rio de Janeiro: PEC/ENSP-ABRASCO; 1987. p. 13-8.

6. Biral AR. Radiações ionizantes para médicos, físicos e leigos. Florianópolis: Insular; 2002.

7. Flôr RC. Exposição ocupacional à radiação ionizante em ambiente hospitalar [dissertação]. Florianópolis: Programa de PósGraduação em Enfermagem, Universidade Federal de Santa Catarina; 2005.

8. Nischimura LY, Potenza MM, Cesaretti IUR. Enfermagem nas unidades de diagnóstico por imagem. São Paulo: Atheneu; 1999.

9. Instituto de PesQuisas Energéticas E Nucleares. Apostila Noções básicas de proteção radiológica. Diretoria de Segurança Nuclear Divisão de Desenvolvimento de Recursos Humanos. [citado em 2 I jan 2005]. Disponível em: http:// www.ipen.gov.br

10. Almeida A, Monteiro AJC, Sakai TK, Pacagnella EZ. Braquiterapia intracavitária na neoplasia uterina. Rev Gaúcha Enferm 1992; 13(2): 42-8.

I I. Peregrino AA F, Almeida CE. Programa de garantia da Qualidade em braquiterapia ginecológica - baixa dose. Nursing 1999; 2(17): 30-4.

12. Haggmark C, Bohman L, Ilmoni-Brandt K, Naslund I, Sjoden PO, Nilsson B. Effects of information supply on satisfaction with information and Quality of life in cancer patients receiving curative radiation therapy. Patient Educ Counseling 2001 ; 45: 173-9.

13. Silva AL. Estudo exploratório de conhecimento dos circulantes de sala de operação sobre radioproteção em centro cirúrgico: subsídio para elaboração de recomendações práticas. In: Anais do $2^{\circ}$ Congresso Brasileiro de Enfermagem em Centro Cirúrgico, 1995. São Paulo (SP), Brasil. São Paulo: SOBECC; 1995. p. 57-60.

14. Diegues SRS, Pires AMT. A atuação do enfermeiro em radioterapia. Rev Bras Cancerol 1997; 43(4).

15. Gonçalves OD, Almeida IPS. Comissão Nacional de Energia Nuclear (RJ) A energia nuclear. Ciência Hoje 2005; 37(220): 44.

16. Silva EC. Dosimetría em radiología intervencionista. In: Anais do International Congress of the International Radiation Protection Association, 2004 May 23-28; Madrid, Espanha. Madrid: IRPA; 2004.

17. Ministério da Ciência e Tecnologia (BR). Comissão Nacional de Energia Nuclear. Resolução CNEN nº. 27/2005. Norma CNEN NN-3.0 I - Diretrizes Básicas de Proteção Radiológica. Brasília: Diário Oficial da União da República Federativa do Brasil; 2005.

18. Ministério da Saúde (BR). Gabinete do Ministro. Portaria $\mathrm{n}^{\circ}$. 198 de 13 de fevereiro de 2004. Minuta Portaria Revisão 1988. Altera a Portaria 198, de 13 de fevereiro de 2004, Que institui a Política Nacional de Educação Permanente em Saúde para adequação às diretrizes operacionais e regulamento do Pacto pela Saúde. Brasília: Ministério da Saúde; 2004. [citado em 7 jul 2007]. Disponível em: http://www.conasems.org.br/files/ MinutaReformulacao 198.pdf

19. Ministério da Saúde (BR). Gabinete do Ministro. Portaria no. 1996 de 20 de agosto de 2007: diretrizes para a implementação da Política Nacional de Educação Permanente em Saúde e dá outras providências. Diário Oficial da União 2007 ag 22.

20. Oliveira ACF, Moreira MC. A enfermagem em radioiodoterapia: Transpondo as barreiras da radioproteção. In: Anais dol $4^{\circ}$. PesQuisando em Enfermagem. 2007 Mai 14- 17; Rio de Janeiro (RJ), Brasil. Rio de Janeiro; EEAN; 2007.

21. Ceccin RB, Ferla AA. Educação Permanente em Saúde. In: Escola Politécnica de Saúde JoaQuim Venâncio; Estação Observatório de Recursos Humanos. Dicionário da Educação Profissional em Saúde. Rio de Janeiro: EPSIV; 2006. p. 7-112.

22. Oliveira SR, Azevedo AC, Carvalho AC. Elaboração de um programa de monitoração ocupacional em radiologia para o Hospital Universitário Clementino Fraga Filho. Radiol Bras 2003.

23. Conselho Federal de Enfermagem (BR). Resolução no. 211 de OI de junho de 1998: atuação dos profissionais de enfermagem Que trabalham com radiação ionizante. Rio de Janeiro: COFEn; 2004. 